\title{
Conceptual design methodology for the preliminary study of a mechatronic system: application to wind turbine system
}

\author{
Ghazoi Hamza ${ }^{1,2,{ }^{*}}$, Moncef Hammadi ${ }^{1}$, Maher Barkallah ${ }^{2}$, Jean-Yves Choley ${ }^{1}$, Alain Riviere ${ }^{1}$, Jamel Louati ${ }^{2}$, and \\ Mohamed Haddar ${ }^{2}$ \\ ${ }^{1}$ QUARTZ EA7393, SUPMECA-Paris, 3 rue Fernand Hainaut, 93407 Saint-Ouen, France \\ 2 Mechanics, Modelling and Production Research Laboratory, National School of Engineers of Sfax, University of Sfax, \\ BP. 1173, Sfax 3038, Tunisia
}

Received: 14 November 2016 / Accepted: 4 April 2017

\begin{abstract}
This paper deals with a methodology for the preliminary design of a mechatronic system regarding the vibrational aspects using analytical approach with Modelica/Dymola. In fact, today, the most commonly used approach for modeling the vibrational behavior of a system is the finite element method. This method is effective however; it is very costly in computation time and space memory and not allows analyzing the whole mechatronic system because of the large number of components to be integrated. The main purpose of this work is to create compact analytical models and especially flexible beams in Modelica/Dymola. These elements can be thereafter inserted in a mechatronic system in order to study its vibrational aspects. The proposed approach aimed to highlight the importance of the structure analysis from the early stage of design and how the developed models allow a fast modeling. Our proposed approach is illustrated for the wind turbine system. In order to predict the motion and the deformation of the flexible system, the derived model considers the structural dynamics of the system and includes the dynamic coupling between the subcomponents. Selected simulation results have been presented to validate the model with respect to scientific reference case. The correctness of the results has been also ascertained by a comparison with those obtained by the finite element analysis using ABAQUS and with those obtained by the model based on the Beam component which belongs to the Modelica Flexible Bodies Library.
\end{abstract}

Keywords: preliminary design / analytical method / Modelica/Dymola

\section{Introduction}

Mechatronic system can be considered as a mechanical, electrical and control subsystem interacting among each other. The mechanical system can be considered as a multiphysic system coupling the mechanical part with other multi-physic parts like thermal, electrical and electromagnetic [1]. The behavior of a mechatronic system should be analyzed by an integrated mechatronic design, taking into account the coupling between the different subsystems [2-4]. Mechatronic systems are confronted with different physical constraints such as temperature and vibration which are in relationship with the system reliability.

The development process of a mechatronic product includes three main phases which are the requirement analysis, the pre-sizing and the detailed sizing. The predimensioning phase of a mechatronic system targets to

\footnotetext{
* e-mail: hamza.ghazoi@gmail.com
}

verify that the chosen design is in accordance with the system requirements and to minimize the risk of new design phase [5-7]. The choices made in this phase will guide all design activities during the detailed design phase [8-10]. To better predict the behavior of a mechatronic system, the pre-dimensioning phase requires the development of robust modeling and simulations of the overall system in order to optimize the search for an optimal design [11].

During the preliminary design, the designers make resort to multi-physics models in order to validate the product numerically, for which the representation of certain physical phenomena is very important [8]. Currently, a major focus is on the development and implementation of numerical methods to simulate physical phenomena. In fact, knowledge of the impact of vibration in the preliminary design of a mechatronic system is rarely considered as essential. Many vibration studies are occurred only after the system manufacturing. When the structure is subjected to dynamic loading caused by forces, it may produce vibration phenomena which are often seen as distracting. 
Wind turbine can be considered as a typical example of a mechatronic system. Such a system operates in a hostile environment. The varying loads with an elastic structure create a perfect breeding ground for induced vibration. Several solutions have been presented to decrease the dynamic loads. As far as the dynamic behavior of a wind turbine is known, we can choose the best shapes and the best material to build it and decrease the maintenance cost. For this reason, it is very important to take a great effort form the early phase of the design cycle of a wind turbine. For instance, a preliminary design of the modular wind turbine tower is proposed in [12]. This approach is based on the study of the rise tower base diameter effects on its tower mass and wall thickness. To validate the model, simplified finite element models have been investigated and comparisons with exact solutions are made. Arbaoui and Asbik [13] proposed a decision support system for the preliminary design of a wind turbine. This method is based on the development of numerous constraints derived from engineering knowledge, to assist various actors in making decisions about choices inherent to their activities in the domain of wind energy. It is targeted to improve performance and decrease the cost of the generated energy.

Jafri et al. [14] developed rotor dynamic model of a wind turbine using an analytical approach. The effects of numerous parameters like rotational speed, wave loading and distribution of mass imbalance on the dynamic response of the system are investigated. A comparison with a three dimensional wind turbine model using the finite element method (FEM) is presented.

Some researchers have been conducted into wind turbine tower structures. For instance, Avila et al. [15] studied the dynamic response of the tower using FEM. A simplified model of the tower was inspected. The system was reduced to a single degree of freedom (SDOF) and the obtained numerical results were compared with those resulting from the analytical solution. Razak [16] developed a wind turbine library in Modelica language. This library provides models of wind turbine performance features such as shaft torque and power output, since Modelica is very appropriate for the establishment of model library [17,18]. The developed model can be applied into any engineering application implying wind turbine technology. The One Wind Modelica library is in development at the Fraunhofer Institute for Wind Energy and Energy System Technology. This library comprises all major components of a wind turbine also models for aerodynamic and hydrodynamic load calculations. The in-house tool One Wind proves how coupled analyses of a wind turbine are realized based on the object oriented modeling language Modelica [19-22]. In an early stage of the design process, simple models are needed to make important design decisions. The main contribution of this work is to propose a method for the preliminary design of a mechatronic system based on an analytical approach, taking into account the effect of vibration. In fact, we developed compact analytical models in Modelica/ Dymola, intended to study the vibrational behavior of a mechatronic system. These models are collected from an exploration of the literature to extract and test the models in the pre-sizing phase of a mechatronic system. In fact,

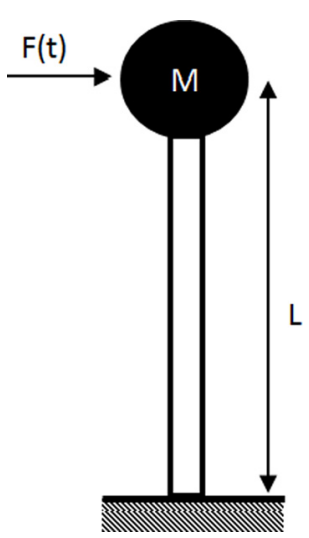

Fig. 1. Flexible beam with a rigid mass.

system architects working with the simulation do not want to spend much time in the implementation of new libraries. Instead, they want to use existing libraries that are recognized as stable and efficient. This approach gives the designer a wide solution space allowing the choice among them. We illustrate this approach with the mechatronical wind turbine system. We established a simple model and a quick method to estimate the dynamic behavior of a mechatronic system using Modelica/Dymola. In this approach, only basic data about the particular wind turbine is required. This paper is structured as follows: the first section deals with this introduction. In Section 2, the theoretical models as well as their implementation in Modelica/Dymola are presented. In Section 3, our methodology is applied to the wind turbine system. Results of simulations are presented and discussed in Section 4. The conclusion of our paper is given in Section 5 .

\section{Development of compact analytical models in Modelica/Dymola}

\subsection{Analytical model of a flexible beam with a rigid mass}

The presented model is concerned with the deflection of the system shown in Figure 1. The system consists of a cantilever flexible beam supporting a rigid mass at its free end [23]. The Euler-Bernoulli beam theory is used to predict deflections. Secondary effects such as shear, axial deformations and rotary inertia are neglected.

The equation of motion governing the transverse vibration of the beam can be formulated as [23]:

$$
E I \frac{\partial^{4} w(x, t)}{\partial x^{4}}+\rho S \frac{\partial^{2} w(x, t)}{\partial t^{2}}=0
$$

The beam has a length $L$, a second moment of the cross sectional area $I, E$ is the Young's modulus, $M$ is the concentrated mass, $M_{t}$ is the beam mass, $\rho$ is the material density and $S$ is the cross-sectional area. We assume that the beam is subjected to an unbalanced force applied to the concentrated mass, through a cyclic force.

The expression of the force is as follows:

$$
F(x, t)=p_{0} \cos (\Omega t)=m e \Omega^{2} \cos (\Omega t)
$$


where $p 0$ is the excitation amplitude, $m$ is the imbalance mass, $e$ is the imbalance radius and $\Omega$ is the rotational speed. Solution (1) must comply with the following boundary conditions:

$$
\begin{aligned}
w(0, t) & =0, \frac{\partial w(0, t)}{\partial x}=0, \frac{\partial^{2} w(L, t)}{\partial x^{2}} \\
& =0,-\left[-E I \frac{\partial^{3} w(L, t)}{\partial x^{3}}\right] \\
& =M \frac{\partial^{2} w(L, t)}{\partial t^{2}}-p_{0} \cos (\Omega t)
\end{aligned}
$$

A separation of variable method is followed to find the solution of equation (1).

$$
w(x, t)=W(x) T(t)=W(x) \cos (\omega t)
$$

By substituting equation (4) into equation (1), we obtain

$$
\frac{W(x)^{(I V)}}{W(x)}-\frac{\rho S}{E I} \Omega^{2}=k^{4}
$$

By assuming the solution of $W(x)$ in the form:

$$
W(x)=A \cos k x+B \sin k x+C \cosh k x+D \sinh k x
$$

where sinh and cosh are hyperbolic functions and $A, B, C$ and $D$ are to be determined from the boundary conditions of the beam with,

$$
k^{2}=\omega \sqrt{\frac{\rho S}{E I}}
$$

The fundamental angular frequency is:

$$
\omega_{1}=\frac{\alpha_{1}^{2}}{L^{2}} \sqrt{\frac{E I}{\rho S}}
$$

where $\alpha_{1}$ is the fundamental eigenvalue. $\alpha_{1}$ depends on the value of the mass ratio $\left(r=\frac{M}{M_{t}}\right)$. Therefore, in order to found the fundamental eigenvalue for any value of the mass ratio $\left(r=\frac{M}{M_{t}}\right)$, we have plotted the curve $\alpha_{1}=f(r)$ (the different values are given in [24]) using Matlab and we looked for an approximate equation (a polynomial of order 8). We obtained the following equation

$$
\begin{aligned}
\alpha_{1} & =3.2 \times 10^{-6} r^{8}-0.00014 \times r^{7}+0.0026 \times r^{6} \\
& -0.025 \times r^{5}+0.15 \times r^{4}-0.5 \times r^{3}+1 \times r^{2} \\
& -13 \times r+1.9
\end{aligned}
$$

Applying the boundary conditions:

$$
\begin{aligned}
W(0) & =0,\left.\frac{\mathrm{d} W(0)}{\mathrm{d} x}\right|_{x=0}=0-\left.E I \frac{\mathrm{d}^{3} W(x)}{\mathrm{d} x^{3}}\right|_{x=L} \\
& =p_{0}+M \Omega^{2} W(L)
\end{aligned}
$$

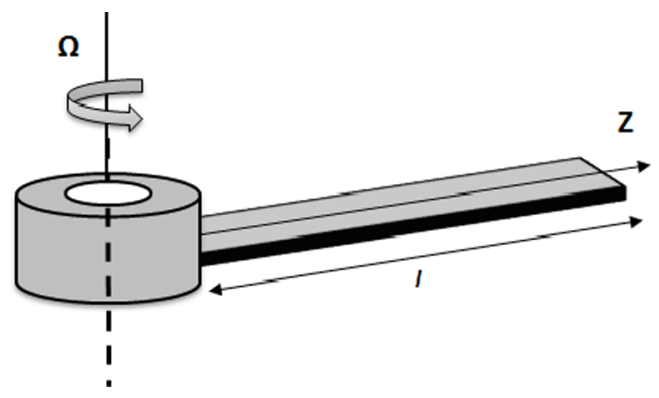

Fig. 2. Flexible rotating beam.

The deflection expression results the following relation for the beam deflection amplitude[23]:

$$
\begin{gathered}
W(x)=-\frac{\frac{p_{0} L^{3}}{E I}}{\beta_{1} \alpha_{1}^{3}\left(\frac{\Omega}{\omega_{1}}\right)^{3 / 2}+\beta_{2} m \alpha_{1}^{4}\left(\frac{\Omega}{\omega_{1}}\right)^{2}} \\
\left(\sin k x-\sinh k x-\frac{\sin k L+\sinh k L}{\cos k L+\cosh k L}(\cos k x-\cosh k x)\right)
\end{gathered}
$$

with,

$$
\begin{gathered}
\beta_{1}=\left(-\frac{\sin k L+\sinh k L}{\cos k L+\cosh k L}(\sin k L-\sinh k L)\right. \\
-\cos k L-\cosh k L)
\end{gathered}
$$

and,

$$
\begin{gathered}
\beta_{2}=\left(\sin k L-\sinh k L-\frac{\sin k L+\sinh k L}{\cos k L+\cosh k L}\right. \\
(\cos k L-\cosh k L))
\end{gathered}
$$

\subsection{Analytical model of a rotating flexible beam}

The rotating beam considered in this paper is assumed to be prismatic cantilever of a length $l$ with rectangular hollow cross-section as illustrated in Figure 2.

Depending on the Euler-Bernoulli theory, the governing differential equation for the rotating beam under flap wise vibration is given by [25]:

$$
\mu(z) \frac{\partial^{2} u}{\partial t^{2}}+\frac{\partial^{2}}{\partial z^{2}}\left(E_{b} I_{b} \frac{\partial^{2} u}{\partial z^{2}}\right)-\frac{\partial}{\partial z}\left(T(z) \frac{\partial u}{\partial z}\right)=p(z, t)
$$

The centrifugal tension force $T(z)$ at a distance $z$ from the origin is given by:

$$
T(z)=\Omega^{2} \int_{z}^{l} y \mu(y) \mathrm{d} y
$$

where $u$ is the relative movement of a point with respect to its static deflected location, $p$ is the applied force per unit length, $\mu(z)$ is the unit mass of the beam per length, $\Omega$ is the rotational speed of the beam, which is assumed to be constant, $E_{b}$ is the modulus of elasticity, $I_{b}$ is the area 


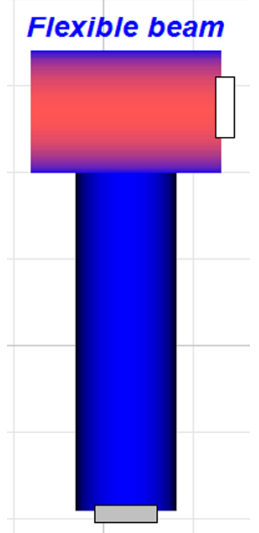

Fig. 3. Icon of the flexible beam model in Modelica/Dymola.

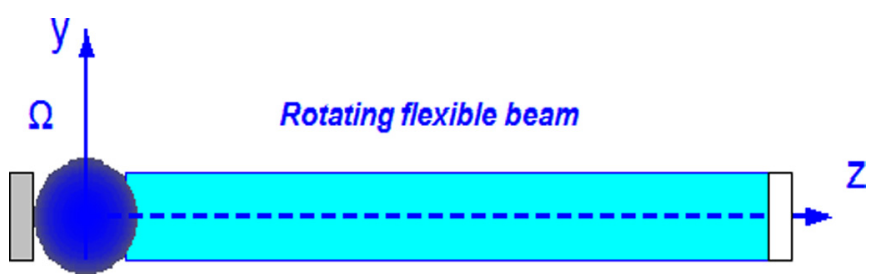

Fig. 4. Icon of the rotating flexible beam model in Modelica/ Dymola.

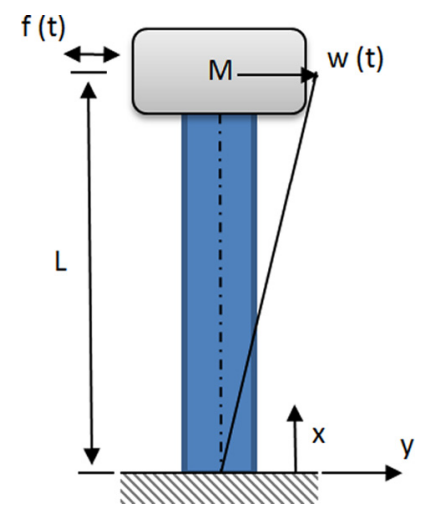

Fig. 5. Preliminary model of a wind turbine.

moment of inertia of the beam cross-section. The impact of gravity on the rotation of the beam is considered to be negligible compared to centrifugal impact.

The clamped and free boundary conditions for the rotating beam are given as follows:

$$
u=\frac{\partial u}{\partial z}=0 \text { at } z=0, \quad \frac{\partial^{2} u}{\partial z^{2}}=\frac{\partial^{3} u}{\partial z^{3}}=0 \text { at } z=l
$$

The mode summation method is considered for the solution which is:

$$
u(z, t)=\sum_{i=1}^{n} \varphi_{i}(z) q_{i}(t)
$$

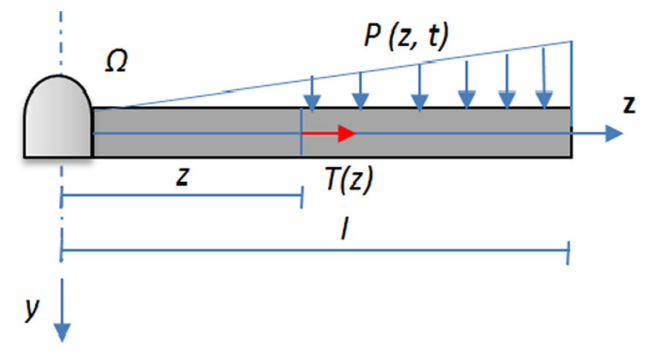

Fig. 6. Preliminary model of a wind turbine blade.

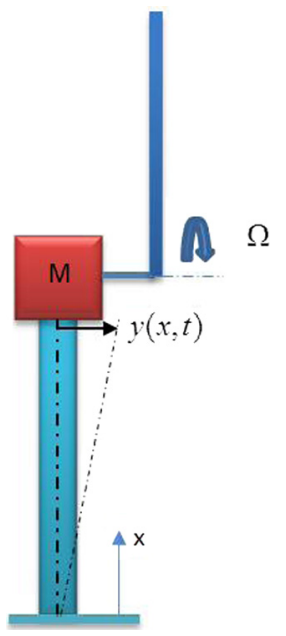

Fig. 7. A simplified model of a coupled tower-blade.

where $\phi_{i}(z)$ are the shapes functions and $q_{i}(t)$ are the generalized coordinates. The shapes functions must be linearly independent and must satisfy the geometric boundary conditions at the hub.

Where $M$ is defined as generalised mass matrix which is:

$$
M=\int_{0}^{l} \mu(z) \phi(z) \phi^{T}(z) \mathrm{d} z
$$

The potential energy of the system is:

$$
U_{d}(q)=\frac{1}{2} q^{T}(t) K q(t)-q^{T}(t) F(t)
$$

where $K$ is the generalized stiffness matrix of the blade which is:

$$
\begin{gathered}
K=K_{e}+K_{g} \\
K_{e}=\int_{0}^{l} E I(z) \frac{\mathrm{d}^{2} \phi(z)}{\mathrm{d} x^{2}}\left(\frac{\mathrm{d}^{2} \phi(z)}{\mathrm{d} z^{2}}\right)^{T} \mathrm{~d} z \\
K_{g}=\int_{0}^{l} T(z) \frac{\mathrm{d} \phi(z)}{\mathrm{d} z}\left(\frac{\mathrm{d} \phi(z)}{\mathrm{d} z}\right)^{T} \mathrm{~d} z
\end{gathered}
$$

$K_{e}$ is the elastic stiffness matrix, $K_{g}$ is the geometric stiffness matrix, $F(t)$ is the dynamic load vector which is computed as:

$$
F(t)=\int_{0}^{l} \phi(z) p(z, t) \mathrm{d} z
$$




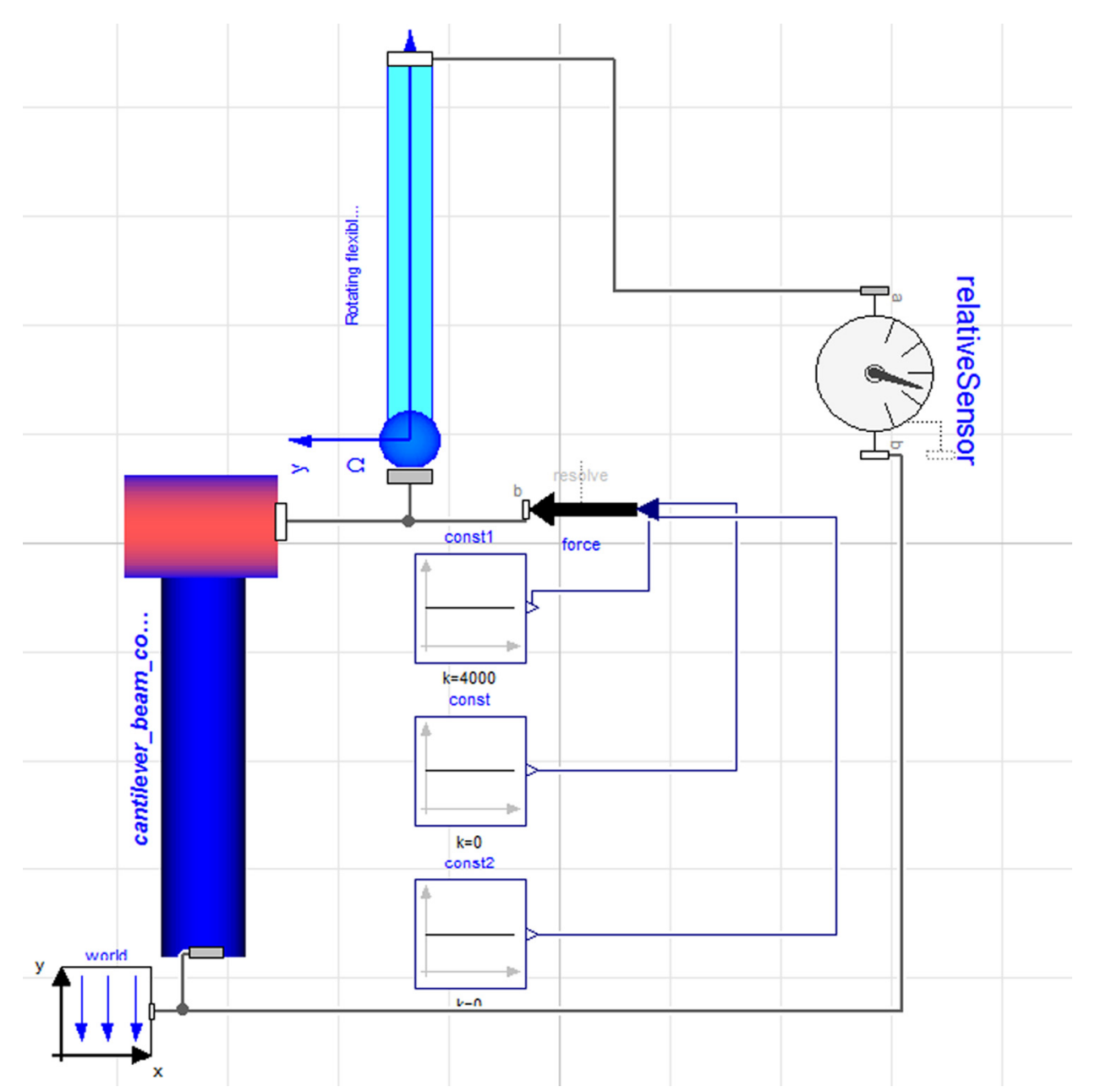

Fig. 8. Preliminary model of the wind turbine system in Modelica/Dymola.

The dynamic model was formulated using the Lagrangian formulation expressed in equation below:

$$
\frac{\mathrm{d}}{\mathrm{d} t}\left(\frac{\partial L}{\partial \dot{q}^{T}}\right)-\frac{\partial L}{\partial q^{T}}=F(t)=-C \dot{q}
$$

where,

$$
\begin{aligned}
L(\dot{q}, q) & =T_{d}(\dot{q})-U_{d}(q) \\
& =\frac{1}{2} \dot{q}^{T} M \dot{q}-\frac{1}{2} q^{T} K q+q^{T} F(t)
\end{aligned}
$$

Substituting this equation into the Lagrangian formulation, we obtain:

$$
M \ddot{q}+C \dot{q}+K q=F(t)
$$

where $\ddot{q}, \dot{q}$ and $q$ are the acceleration, velocity and displacement vector; $C$ is the damping matrix.

The beam was considered to be vibrating in its fundamental mode and a quadratic mode shape was assumed. This allowed the reduction of the continuous beam to a SDOF. Beam bending motion in the ' $y z$ ' plan is only of interest, where this motion is termed flapping motion.
The relative displacement of the beam $u(z, t)$ can be expressed as

$$
u(z, t)=\phi(z) q(t)
$$

The shape function is taken as:

$$
\phi(z)=\frac{3}{2}\left(\frac{z}{l}\right)^{2}\left(1-\frac{1}{3} \frac{z}{l}\right)
$$

The generalised mass matrix and stiffness matrix are then

$$
M=\frac{33}{140} \mu l, \quad K_{e}=3 \frac{E I}{l^{3}}, \quad K_{g}=\frac{81}{280} \mu l \Omega^{2}
$$

The angular eigen-frequency at $\Omega=0$ can be expressed as:

$$
\omega_{1,0}=\sqrt{\frac{K_{e}}{M}}=\sqrt{\frac{140}{11}} \sqrt{\frac{E I}{\mu l^{4}}}
$$




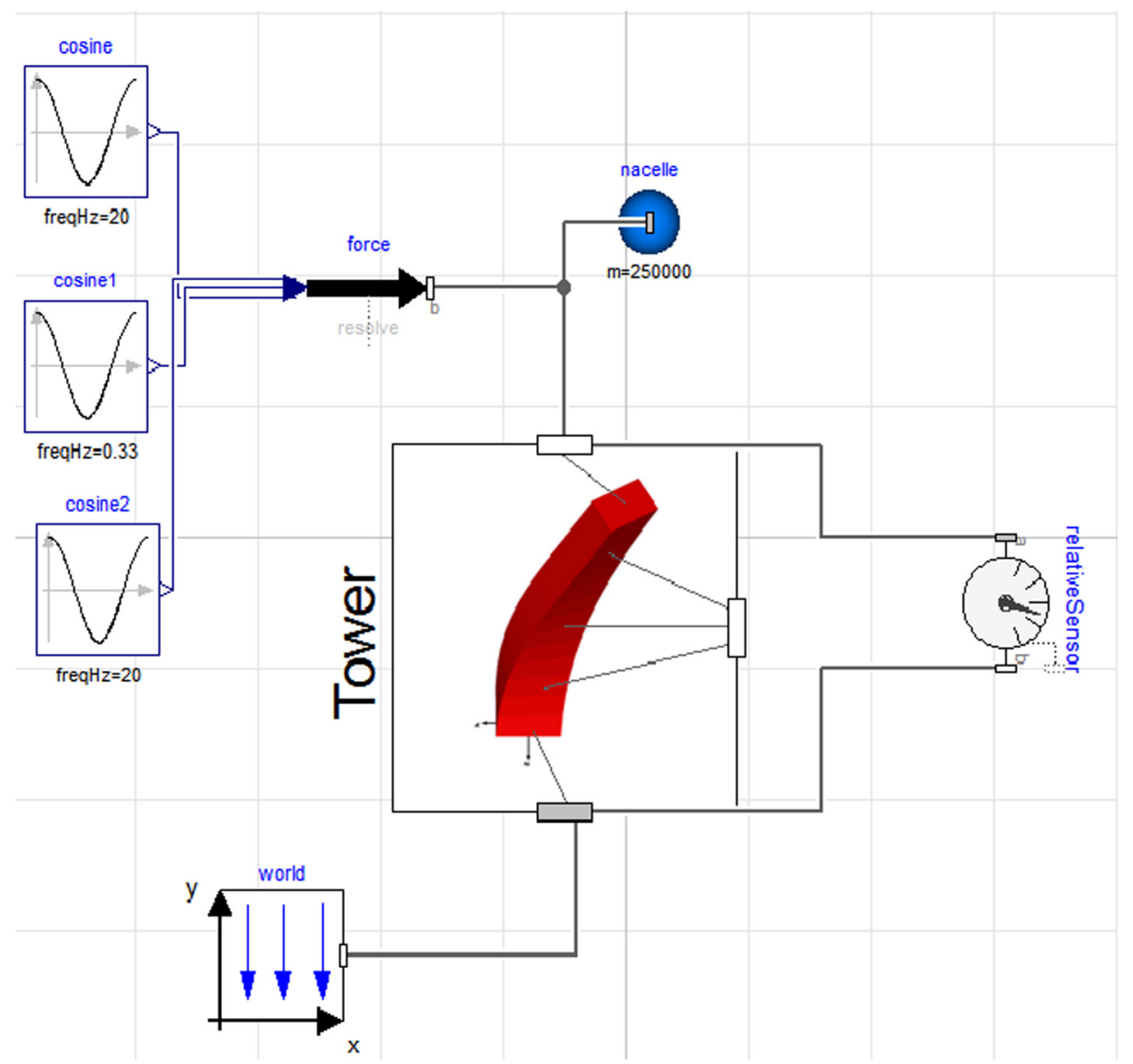

Fig. 9. Set-up of the wind turbine model deflection.

And, the angular frequency at $\Omega>0$ becomes

$$
\begin{aligned}
\omega_{1} & =\sqrt{\left(\frac{K_{e}}{M}+\frac{K_{g}}{M}\right)}=\omega_{1,0} \sqrt{\left(1+\frac{K_{g}}{K_{e}}\right)} \\
& =\omega_{1,0} \sqrt{\left(1+\frac{27}{22} \frac{\Omega^{2}}{\omega_{1,0}^{2}}\right)}
\end{aligned}
$$

We consider that the applied load is a harmonic triangular distributed per unit with the frequency of this sinusoid being equal to the rotational frequency as shown in Figure 2. This load may be expressed as:

$$
p(z, t)=p_{0}(t) \frac{z}{l}=p_{0} \cos (\Omega t) \frac{z}{l}
$$

For zero initial condition and without taking into account the damping effect, the steady-state analytical solution is then:

$$
\begin{aligned}
u(z, t) & =\frac{11 \cdot l \cdot p_{0}}{40 \cdot M\left(\omega_{1}^{2}-\Omega^{2}\right)} \cos (\Omega t) \\
& \times \frac{3}{2}\left(\frac{z}{l}\right)^{2}\left(1-\frac{1}{3} \frac{z}{l}\right)
\end{aligned}
$$

\section{Implementation of the flexible structures in Modelica/Dymola}

In this part, the models described above were implemented in Modelica/Dymola, since Modelica is adequate for the models library elaboration. The analytical model of the flexible beam with a rigid mass has been implemented in Modelica/Dymola, taking into account the essential equations describing the bending vibration of the structure, creating so a new component called "Flexible beam" (Fig. 3). The model has been implemented with two connectors which are "Frame a" and "Frame b". The choice of the connector "Frame" makes the object "Flexible beam" compatible with other components existing in Modelica library and then, it can be inserted in different mechatronic systems, as it is allowed by the object oriented structure of the Modelica language. The rotating flexible beam has also been modeled in Modelica/Dymola creating so an objected called "Rotating flexible beam". Figure 4 shows the "Rotating flexible beam" icon developed in Modelica/ Dymola. This model was implemented with two connectors Frames (Frame $a$ and Frame b). This element includes the geometric and the materiel properties of the beam and the load characteristics which can be changed by the user by modifying its respective parameter window. 
Table 1. Parameters of the wind turbine model.

\begin{tabular}{ll}
\hline Parameter & Values \\
\hline Tower outside diameter & $4.5720 \mathrm{~m}$ \\
Tower thickness $(e)$ & $0.09525 \mathrm{~m}$ \\
Tower height $(h)$ & $91.5 \mathrm{~m}$ \\
Nacelle mass $(M)$ & $200000 \mathrm{~kg}$ \\
Blades mass $(m)$ & $50000 \mathrm{~kg}$ \\
Rotor imbalance & $0.05 \mathrm{~m}$ \\
Rotational speed & $20 \mathrm{tr} \mathrm{min}^{-1}=2.08 \mathrm{rad} \mathrm{s}^{-1}$ \\
Steel density & $7850 \mathrm{~kg} \mathrm{~m}^{-3}$ \\
Elastic modulus $(E)$ & $206841.9 \times 10^{6} \mathrm{~N} \mathrm{~m}^{2}$ \\
\hline
\end{tabular}

\section{Application to the wind turbine system}

\subsection{Preliminary model of a wind turbine}

The wind turbine structure is idealized by replacing it with an assembling of a flexible tower and a rigid mass at the top representing the nacelle and the blades[26]. It will be assumed that the tower behaves as a cantilever beam of circular hollow cross-section, fixed at the ground. The nacelle and the rotor blades will be combined as one mass at the beam end. The tower is subjected only to a load which represents the effect of rotor imbalance (Fig. 5). The model of the Flexible Beam which was developed in Modelica/ Dymola (Fig. 3) can represent well a preliminary model of the wind turbine. In fact, this model allows us to have a first insight about the dynamic behavior of a wind turbine subjected to load which depicts the effect of the rotor imbalance.

\subsection{Preliminary model of the wind turbine blade}

The wind turbine blade is the most flexible component of this machine. The aerodynamic loads acting on the blade are divided into two components: loads in the rotor plane and loads out of the rotor plane. The loads out of the rotor plane do not contribute to the generation of the mechanical power, but constitute the largest part of the mechanical loading on the blade. In this study, we consider only the loads out of the rotor plane (Fig. 6) and then the blade flapwise bending. So, the Rotating flexible beam model developed in Modelica/Dymola (Fig. 4) is well suited for modeling the wind turbine blade subjected to transverse vibrations due to the wind loading.

\subsection{Description of the coupled tower-blade system}

The model of a coupled tower-blade is illustrated in Figure 7. In fact, a point mass $M$ representing the nacelle is attached to the tip of the tower and a blade rotating at speed $\Omega$. The blade and the tower are considered to be Euler-Bernoulli beams with homogeneous and uniform cross sections. The rotor is subjected to a wind thrust force which is in turn, transferred through the nacelle compo-

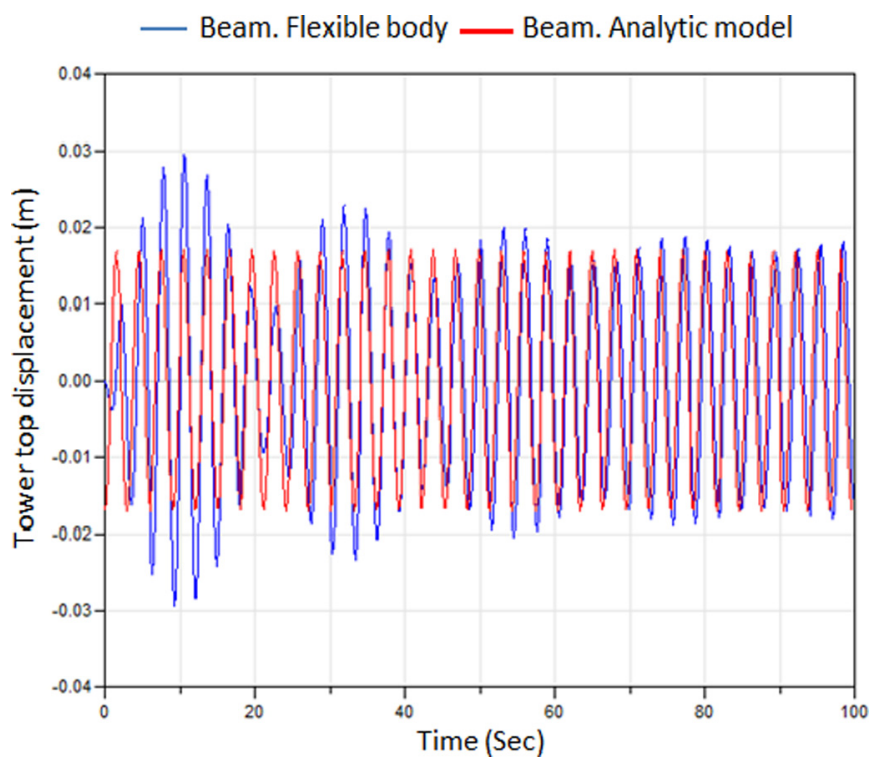

Fig. 10. Bending amplitude of the beam.

nents to the top of the tower. The deformation of the blade and the tower happens in the same plan. The blade is subjected to a transverse motion of the base due to the nacelle vibration (the blade is fixed on the nacelle which is in turn, fixed to the end-tip of the tower) and to a normal force which represents the projection of the aerodynamic force to the rotor plan. This model allows studying the dynamic interaction between the tower and the blade. Each of components described previously (Flexible beam and Flexible rotating beam) includes two frame connectors from the Multibody library in order to connect adjacent components of the wind turbine system and then, allow the interaction between the subcomponents (the tower and the blade). Thus, it is simply possible to integrate improved models for subcomponents using parts of the Modelica Standard Library. Figure 8 shows the model of a coupled tower-blade developed in Modelica/Dymola. This model contains a set of connected elements. Elements which are created in Modelica/Dymola such as the Flexible beam and the Rotating flexible beam and elements that belong to Modelica library such as sensor to measure the absolute position, world reference system and signals input to apply external forces. This model will provide us the opportunity to study the dynamic interaction between the tower and the blade when the top of the tower (nacelle) is subjected to the wind thrust force and the blade is subjected to aerodynamic load.

\section{Numerical results}

\subsection{Validation of the compact analytical models}

\subsubsection{Model validation of the flexible beam with a rigid mass}

In order to validate the dynamic behavior of the wind turbine (Flexible beam), some results derived in this paper were compared with those obtained using the Beam component which belongs to Modelica flexible Library. The developed model (Fig. 9) mainly consists of a world reference system to define the gravity direction, a flexible 
Table 2. Parameters of the wind turbine blade.

\begin{tabular}{ll}
\hline Parameter & Value \\
\hline Blade length & $30 \mathrm{~m}$ \\
Blade width & $2.8 \mathrm{~m}$ \\
Blade depth & $0.8 \mathrm{~m}$ \\
Blade thickness & $0.01 \mathrm{~m}$ \\
Rotational frequency of the blade & $1.57 \mathrm{rad} \mathrm{s}^{-1}$ \\
Blade Young's modulus & $6.5 \times 10^{11} \mathrm{~N} \mathrm{~m}^{2}$ \\
Materiel density & $2100 \mathrm{~kg} \mathrm{~m}^{-3}$ \\
Inertia moment in the flapping sense & $9.53 \times 10^{-3} \mathrm{~m}^{4}$ \\
\hline
\end{tabular}

beam named "Tower" belonging to Modelica flexible library and a concentrated mass at the tip of the beam, depicts the mass of the nacelle and the rotor blades.

The beam is clamped at one end and a harmonic excitation force is applied to the other end, which represents the effect of imbalance. A relative sensor is used to predict the motion of the nacelle according to its base. Table 1 summarizes the parameters used for the simulation. Figure 10 shows a comparison of the nacelle vibration amplitude between the two models. The first model using the beam compact model based on the developed analytical approach and the second one using a beam which belongs to the flexible library of Modelica. From the presented results, it can be observed that the two curves have a sinusoidal shapes and a same frequency as that of the excitation. For the model based on the beam belonging to the flexible library of Modelica, the vibration amplitude at the beginning is variable. So, the excitation influences hardly the state of the beam. Subsequently, the vibration amplitude becomes stable and the two curves have nearly the same amplitude of vibration. The two models are then close to each other.

\subsubsection{Model validation of the rotating flexible beam}

In order to validate the flexible rotating beam model implemented in Dymola/Modelica, the results derived in this paper have been compared with those obtained with the finite element analysis using ABAQUS (dynamic analysis). The physical and mechanical properties of the analyzed beam [27] are listed in Table 2 .

Table 3 contains the first six natural frequencies obtained by ABAQUS. The first natural frequency determined by FEM analysis is equal to $4.015 \mathrm{~Hz}$ and that obtained by Dymola (analytical result) is equal to $4.04 \mathrm{~Hz}$. Results are then very close. A comparison between the tip deflections of a rotating flexible beam models is shown in Figure 11. The first model is based on FEM (ABAQUS) and the second model is based on the analytical method developed in Modelica/Dymola. It can be seen that the two curves have the same frequency which is equal to the excitation frequency $(0.25 \mathrm{~Hz})$ and they have nearly the same vibration amplitude. These results can validate our approach.
Table 3. Natural frequencies of the beam obtained by ABAQUS.

\begin{tabular}{lc}
\hline Mode & Value $(\mathrm{Hz})$ \\
\hline 1 & 4.015 \\
2 & 41.39 \\
3 & 52.81 \\
4 & 146.63 \\
5 & 463.19 \\
6 & 1436.6 \\
\hline
\end{tabular}

\subsection{Parametric study}

After modeling the wind turbine substructures with Modelica/Dymola, we performed a parametric analysis. Several simulation analyzes have been performed in order to study the impact of various parameters taken into account by our preliminary model on the vibration behavior of wind turbine. The vibration amplitudes of some points of the wind turbine are shown in Figure 12. As it can be observed, the maximum bending is at the nacelle level and the minimum is at the tower base $(x=0)$ which is well adequate with the boundary condition (the tower is fixed to the ground). Moreover, all positions show their responses to be in phase with each other and they have the same frequency as that of the excitation. The results show a good agreements with those obtained in [14] for the same geometrical characteristics. Therefore, regardless parameters we can control vibration at any point of the wind turbine. The imbalance imposed at the nacelle through the harmonic excitation has a great impact on the dynamic response. Figures 13 and 14 illustrate the nacelle deflection according respectively, to the rotor blades effective eccentricity and to the combined rotor blades mass. As it can be seen, the bending increases with increasing the excitation amplitude. Therefore, the rotor characteristics have a significant impact on the dynamic response of the wind turbine.

Figure 15 illustrates the nacelle deflection according to the rotor rotational speed. From the presented result, a typical resonance position is occurred for the rotational speed value of the order of $2.3 \mathrm{rad} \mathrm{s}^{-1}$. This value is well corresponding to the fundamental angular frequency of the system. An analysis of the blade response has been also performed. We used specifications listed in Table 2. Figure 16 shows the computed blade displacements time history for $\Omega=1.57 \mathrm{rad} \mathrm{s}^{-1}$. It can be seen that displacements have sinusoidal shapes. The maximum deformation takes place at the blade tip and the minimum is at the hub level. After analyzing the dynamic behavior of the wind turbine sub-components (tower and blade), the bladetower coupling effect was investigated. The motion of the tower and the blade is coupled by means of the interactive force at the nacelle which represents the wind thrust force and the aerodynamic force which is applied to the blade. The relative and the absolute tip vibration of the blade are shown in Figure 17. It was observed that the total displacement of the blade is the sum of the blade displacement relative to the nacelle and the deflection at the tower top relative to the ground. 


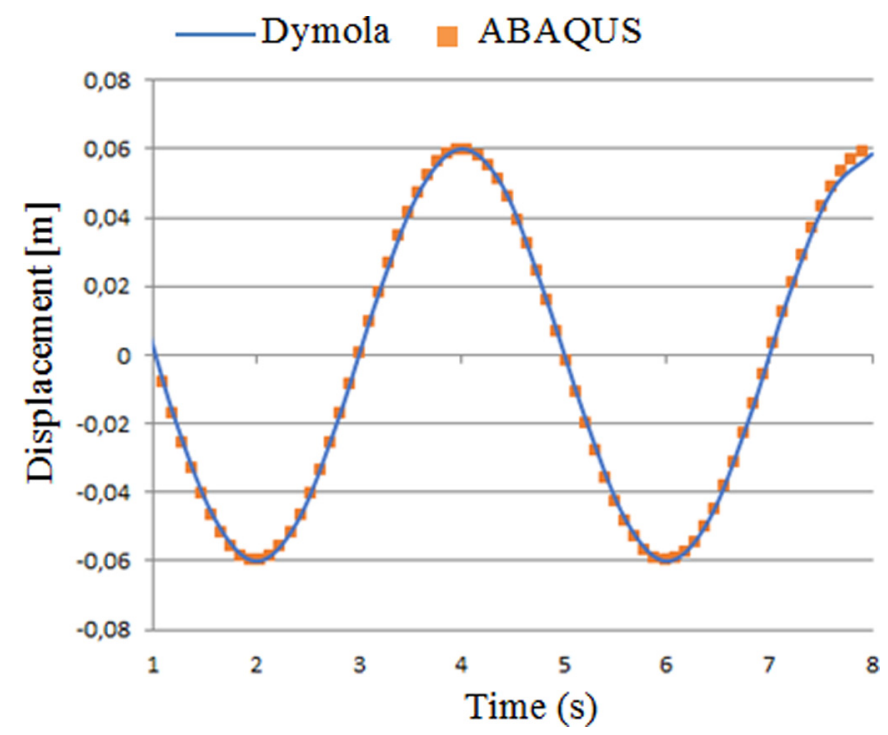

Fig. 11. Tip vibration of the blade comparison.

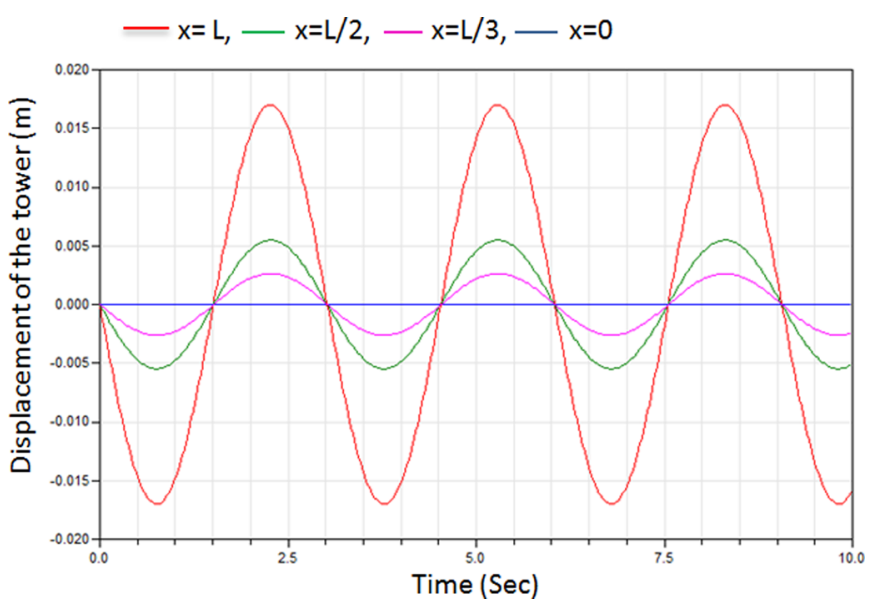

Fig. 12. Displacement of the wind turbine at different points analytical solution.

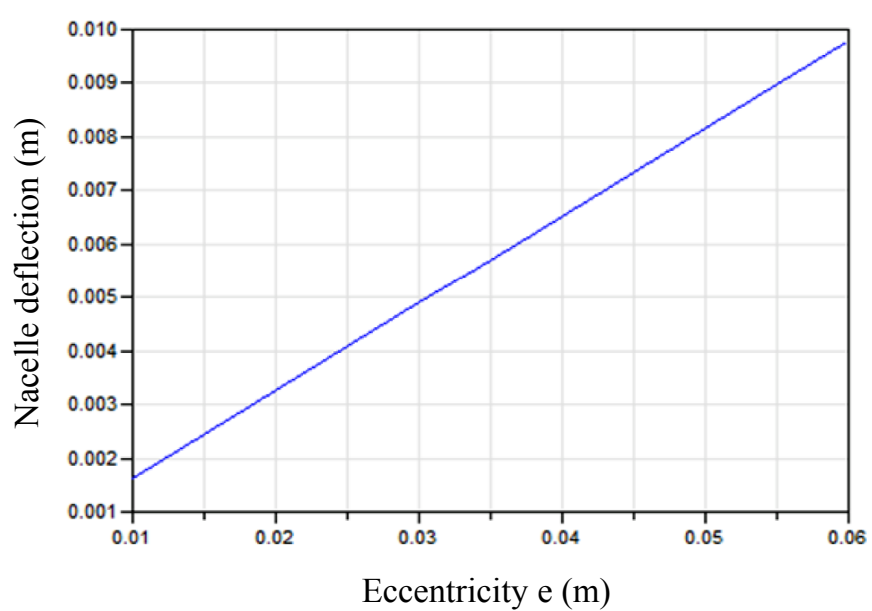

Fig. 13. Nacelle deflection according to the blades eccentricity.

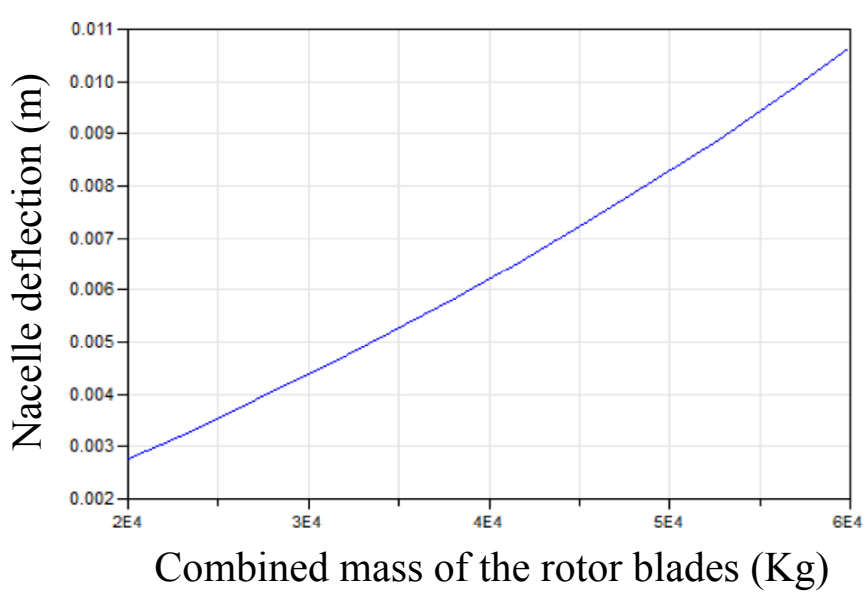

Fig. 14. Nacelle deflection according to the rotor mass.

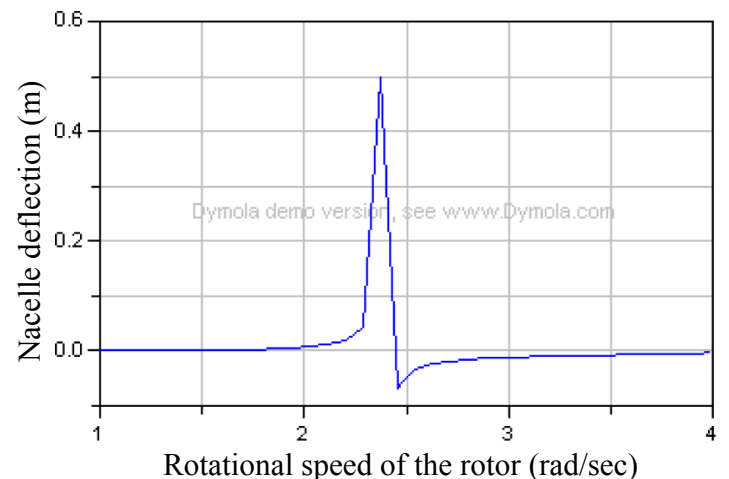

Fig. 15. Nacelle deflection according to the rotor rotational speed.

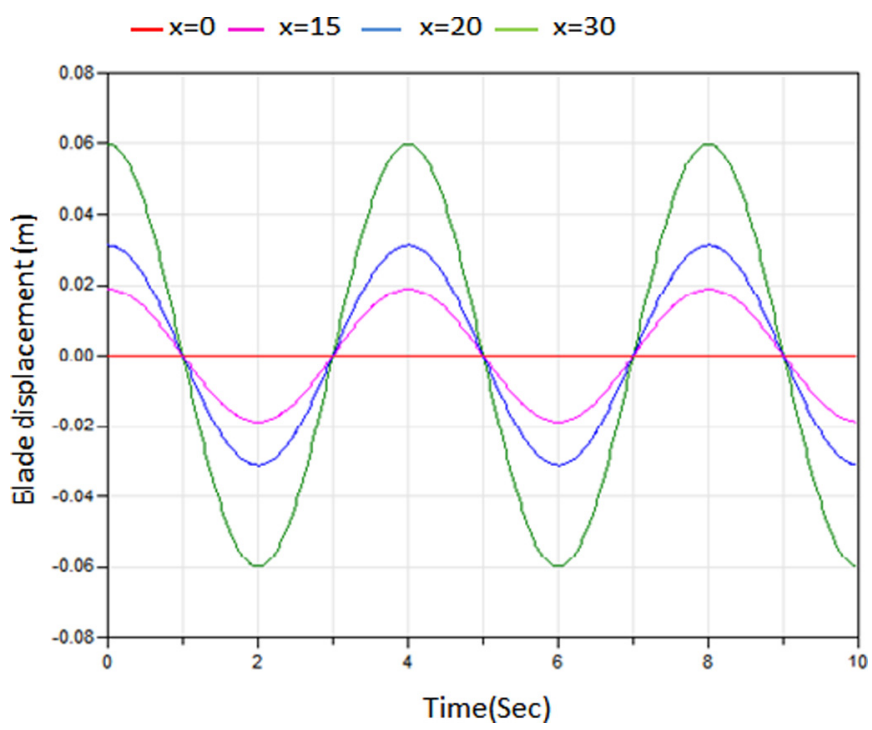

Fig. 16. Tip displacement response of the blade with $\Omega=$ $1.57 \mathrm{rad} \mathrm{s}^{-1}$. 


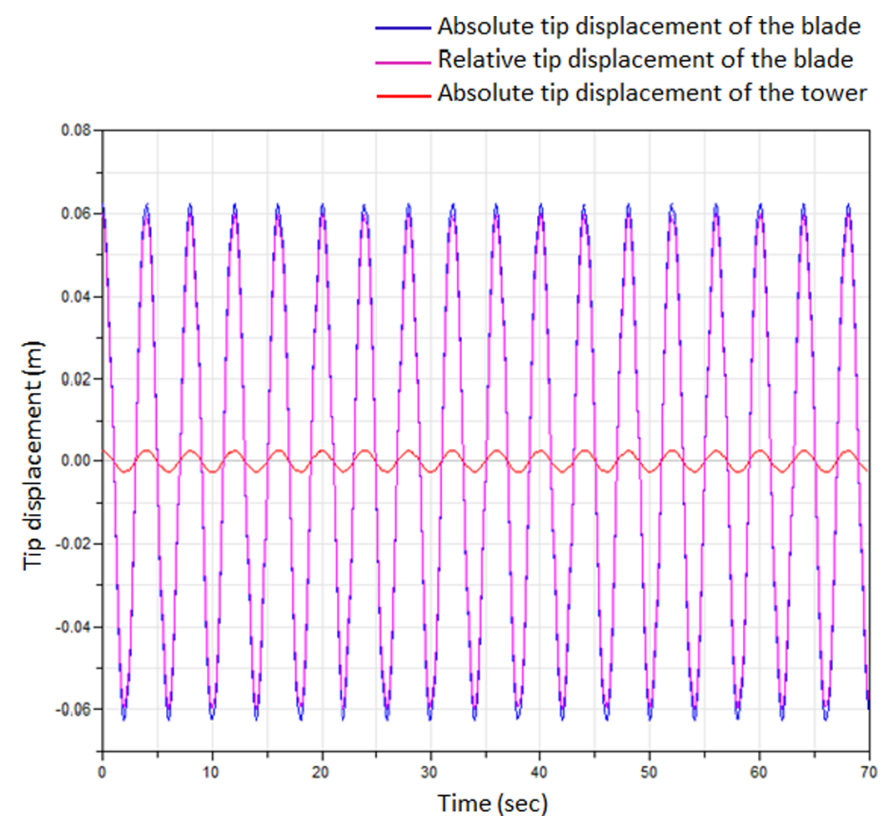

Fig. 17. Tip displacement.

\section{Conclusion}

In this paper, a new methodology for the preliminary design of a mechatronic system has been developed, based on analytical approach. In fact, a models library formed by two flexible beam models has been developed in Modelica/ Dymola based on analytical methods. These elements can be furthermore inserted in a mechatronic system in order to study its vibrational response in an early stage of the design cycle. Having a methodology that allows designers to create models with high level of accuracy, from the early stage of design, will ameliorate the choice of the initial ideas and decrease time of the design cycle. We illustrate this methodology to the case of a wind turbine system in order to have an insight about its dynamic behavior. The impact of different parameters on the system response is highlighted. Due to the object-oriented structure of Modelica, the different components required for the system simulation can be implemented independently and then allows studying the dynamic interaction between the system subcomponents. This modelling process will support designers to quickly build up system models, the analysis, and the characterization of complex system which is formed by distributed and discrete components. The presented work can be a useful tool for the verification and the perspicacity into the dynamic response of a mechatronic system before engaging in advanced and detailed modeling.

\section{References}

[1] M. Hammadi, J.Y. Choley, F. Mhenni, A multi-agent methodology for multi-level modeling of mechatronic systems, Adv. Eng. Inform. 28 (2014) 208-217
[2] M. Hammadi, J.Y. Choley, O. Penas, A. Riviere, J. Louati, M. Haddar, A new multi-criteria indicator for mechatronic system performance evaluation in preliminary design level, in: 13th International Workshop on Mechatonics, 9th France-Japan and 7th Europe-Asia Congress on Research and Education in Mechatronics (REM), 2012, pp. 409-416

[3] M. Hammadi, J.Y. Choley, Parametric compact modelling of dynamical systems using meshfree method with multi-port technique, Int. J. Dyn. Syst. Diff. Eq. 5 (2015) 206-219

[4] P. Lisandrin, M. van Tooren, High-order finite elements reduced models for modal analysis of stiffened panels, Int. J. Mech. Mater. Des. 3 (2006) 111-127

[5] G. Hamza, J.Y. Choley, M. Hammadi, M. Barkallah, J. Louati, A. Riviere, M. Haddar, Pre-designing of a mechatronic system using an analytical approach with dymola, J. Theor. Appl. Mech. 53 (2015) 697-710

[6] G. Hamza, J.Y. Choley, M. Hammadi, M. Barkallah, J. Louati, A. Riviere, M. Haddar, Pre dimensioning of the dynamic properties of the wind turbine system using analytical approach, in: Proceedings of the Sixth Conference on Design and Modeling of Mechanical Systems (CMSM '2015), Hammamet, Tunisia, 2015

[7] M. Hammadi, J.Y. Choley, O. Penas, A. Riviere, A multidisciplinary approach for modelling and optimization of road electric vehicles in conceptual design level, in: IEEE International Conference on Electrical Systems for Aircraft, Railway and Ship Propulsion, Aachen, Germany, 2012

[8] G. Rzevski, On conceptual design of intelligent mechatronic systems, Mechatronics 13 (2003) 1029-1044

[9] L. Wang, W. Shen, H. Xie, J. Neelamkavil, A. Pardasani, Collaborative conceptual design - state of the art and future trends, Comput. Aided Des. 34 (2012) 981-996

[10] T. Habib, H. Komoto, Comparative analysis of design concepts of mechatronics systems with a CAD tool for system architecting, Mechatronics 24 (2014) 788-804

[11] G. Hamza, J.Y. Choley, M. Hammadi, M. Barkallah, J. Louati, A. Riviere, M. Haddar, Analytical approach for the integrated preliminary analysis of mechatronic systems subjected to vibration, Mecatronics, in: 10th FranceJapan/8th Europe-Asia Congress, Tokyo, Japan, 2014

[12] C. Chantharasenawong, P. Jongpradist, S. Laoharatchapruek, Preliminary design of 1.5-MW modular wind turbine tower, in: The 2nd TSME International Conference on Mechanical Engineering, Krabi, Thailad, 2011

[13] A. Arbaoui, M. Asbik, Constraints based decision support for site-specific preliminary design of wind turbines, Energy Power Eng. 2 (2010) 161-170

[14] S.M. Jafri, A. Eltaher, P. Jukes, Dynamics of offshore wind turbines, in: Proceedings of the Twenty-first International Offshore and Polar Engineering Conference, ISOPE, Hawaii, USA, 2011, pp. 19-24

[15] S.M. Avila, M.A.M. Shzu, M.W. Pereira, L.S. Santos, M.V. G. Morais, Z.J.G. Prado, Numerical modeling of the dynamic behavior of a wind turbine tower, in: 11th International Conference Vibration Problems, Lisbon, Portugal, 2013

[16] A.A. Razak, Overview of wind turbine modeling in Modelica, Int. J. Eng. Technol. 4 (2012) 551-553

[17] A.B. Dynasym, Dymola dynamic modeling laboratory user's manual, 2006. Available from: http://www.dymola.com/

[18] Modelica Association, Modelica - A Unified Object-Oriented Language for Systems Modeling v3.3. Standard Specification, 2012 
[19] M. Strobel, F. Vorpahl, C. Hillmann, X. Gu, A. Zuga, U. Wihlfahrt, The one wind modelica library for offshore wind turbines implementation and first results, in: Proceedings 8th Modelica Conference, Dresden, Germany, 2011

[20] M. Brommundt, M. Muskulus, M. Strach, M. Strobel, F. Vorpahl, Experiences with object-oriented and equation based modeling of a floating support structure for wind turbines in Modelica, in: Proceedings of the 2012 Winter Simulation Conference, Berlin, Germany, 2012

[21] R. Samlaus, P. Fritzson, A. Zuga, M. Strobel, C. Hillmann, Modelica code generation with polymorphic arrays and records used in wind turbine modeling, in: Proceedings of the 9th International Modelica Conference, Munich, Germany, 2012

[22] C. Rickert, Object-oriented modeling of a wind power plant in modelica and analysis of loads on blade bearings, Master's thesis, School of Industrial Engineering and Management (ITM), Energy Technology, Brinellvägen, Stockholm, 2012
[23] D.V. Bambill, S.J. Escanes, C.A. Rossit, Forced vibrations of a clamped-free beam with a mass at the free end with an external periodic disturbance acting on the mass with applications in ships structures, Ocean Eng. 30 (2003) 1065-1077

[24] P. Laura, J. Pombo, E. Susemihl, A note on the vibrations of a clamped-free beam with a mass at the free end, J. Sound Vib. 37 (1974) 161-168

[25] J. Arrigan, V. Pakrashi, B. Basu, S. Nagarajaiah, Control of flapwise vibrations in wind turbine blades using semi-active tuned mass dampers, Struct. Control Health Monit. 18 (2011) 840-851

[26] H. Robert, H. Erich, S. Herman, Large wind turbines: design and economics, John Wiley \& Sons, Inc., London, 2000

[27] P.J. Murtagh, B. Basu, B.M. Broderick, Along-wind response of a wind turbine tower with blade coupling subjected to rotationally sampled wind loading, Eng. Struct. 27 (2005) 1209-1219

Cite this article as: G. Hamza, M. Hammadi, M. Barkallah, J.-Y. Choley, A. Riviere, J. Louati, M. Haddar, Conceptual design methodology for the preliminary study of a mechatronic system: application to wind turbine system, Mechanics \& Industry 18, 413 $(2017)$ 